\title{
Direct Alcohol Fuel Cells as an Enabling Clean Energy Technology for Portable Devices and Transportation - From Fundamental to System Engineering
}

\author{
Wen-Feng Lin \\ Department of Chemical Engineering, Loughborough University \\ Loughborough, Leicestershire, LE11 3TU, England, UK. \\ W.Lin@lboro.ac.uk
}

Direct Alcohol Fuel Cells (DAFCs), as an emerging new clean energy technology, are very attractive as power sources for mobile and portable applications. Nonetheless, on the basic research side, a significant challenge is to gain a fundamental understanding of fuel cell nanocatalyst structures and their corresponding catalytic reaction mechanisms. The fundamental studies can provide a platform not only for understanding nanocatalyst performance but also for exploring the structureactivity relationship at atomic and molecular level; and ultimately for rationally designing new nanocatalysts. On application side, innovation in polymer electrolyte materials, single cell and stack design is crucial for achieving high power density and developing efficient fuel cell systems as a versatile clean energy technology.

First part of this talk is on fundamental nanomaterials and electrocatalysis studies. A series of low cost and high performance Pd based and low loading Pt based nanocatalysts supported on various substrates such as WC have been developed. The surface structure and reactivity of the prepared nanocatalysts towards the electro-oxidation of a range of small organic fuel molecules in various electrolyte solutions have been studied by combined in-situ electrochemical FTIR spectroscopy and Density Functional Theory calculations. New insights into the surface structures and electrocatalysis have been obtained at atomic and molecular level. The electro-catalytic activity of nanocrystals/nanocatalysts is highly dependent on both the composition and the surface morphology, largely attributing to electronic effect, surface atomic arrangement and coordination. For an example, modified Pd tetrahexahedral nanocrystals (NCs) with Bi decoration were prepared and their reactivity towards ethanol oxidation reaction (EOR) has been studied in alkaline media at various temperatures and under practical DAFC operation conditions.

Second part of this talk is on fit-for-purpose fuel cell system development, novel electrode structure, new polymer electrolyte, innovative single cell and stack design and fabrication will be reported.

\section{Acknowledgment}

Financial support from the UK EPSRC (EP/I013229/1, TS/H001875/1, EP/K014706/1) is gratefully acknowledged. 